\title{
Supplementary Material: Sensitivity of the southern hemisphere tropospheric jet response to Antarctic ozone depletion: prescribed versus interactive chemistry
}

\section{Model Bias Reduction}

Table 2 showed the maximum polar cap temperature trend in the SH lower stratosphere in our model simulations and in the WACCM-CCMI simulations published in Calvo et al. (2017). The different trend between our Chem ON ensemble $\left(-6.6 \pm 1.4 \mathrm{~K}_{\text {decade }}{ }^{-1}\right)$ and the WACCMCCMI ensemble $\left(-4.4 \pm 2.8 K\right.$ decade $\left.^{-1}\right)$ is most probably due to the fact that we did not implement all of the changes to the gravity wave parameterizations as done in WACCM-CCMI. According to Garcia et al. (2017) the gravity wave parametization changes are crucial for the bias correction. Furthermore, in WACCM-CCMI some changes to the chemistry scheme were introduced that are also not implemented in the WACCM version used here. Another difference can be found in the solar forcing used in our simulations. Here, we made use of the new CMIP6 solar forcing, while the WACCM-CCMI version uses the CMIP5 solar forcing. These two forcings show some differences in the total solar irradiance but also specifically in the UV-bands: the CMIP6 forcing exerts substantially larger variability in the solar cycle for the 200-400 nm range, especially showing higher values under solar maximum conditions (Matthes et al., 2017).

Apart from the differences in the model versions, we used an ensemble of 9 members, whereas Calvo et al. (2017) used only 3 ensemble members. To a certain extent, the different maximum trends shown in Table 2 could therefore also be influenced by internal variability and the different amount of data available to calculate the trends.

Figures 1 and 2 show the impact of the adaptations made to the model code as described in the methods section in a pre-industrial control setting. In the annual mean (Fig. 1) the modifications lead to an increase of the SH polar cap temperatures by $2.5 \mathrm{~K}$ in the middle stratosphere and to a reduction of the strength of the stratospheric polar night jet (PNJ) by about $9 \mathrm{~ms}^{-1}$. These differences are larger for the austral winter and spring seasons with an increase in polar temperatures by 5.4 and $4.9 \mathrm{~K}$ and a decrease in the strength of the PNJ by 15.0 and $10.3 \mathrm{~ms}^{-1}$ respectively. 

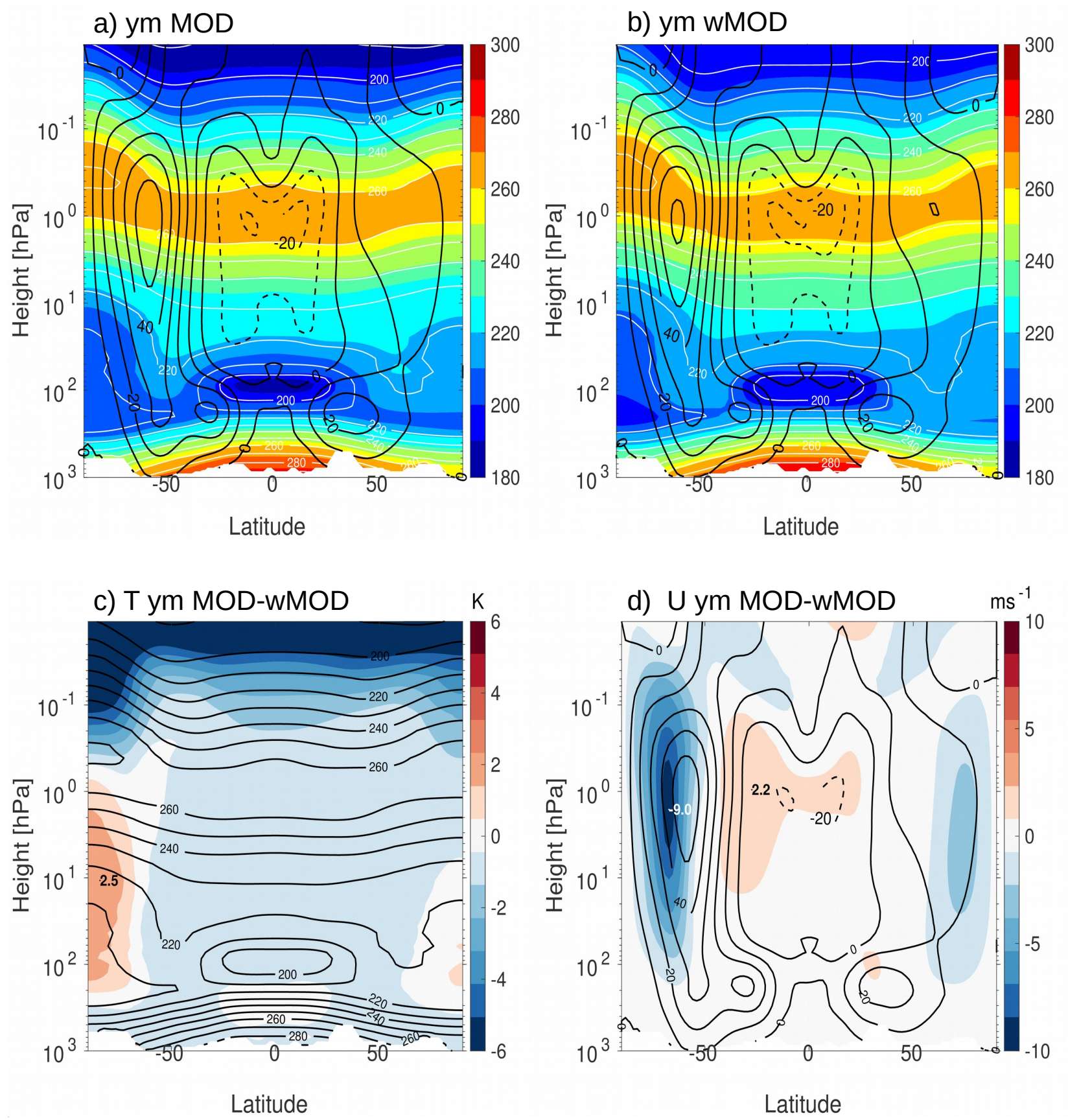

Figure 1: Annual mean zonal mean temperature in K (shading) and zonal mean zonal wind in $m^{-1}$ (contours) in a pre-industrial control simulation (a) with model code adjustments (MOD) and (b) without model code adjustments (wMOD) as described in the methods section and the difference between MOD and wMOD (shading) along with the mean for MOD (contours) for temperature (c) and zonal wind (d) respectively. 

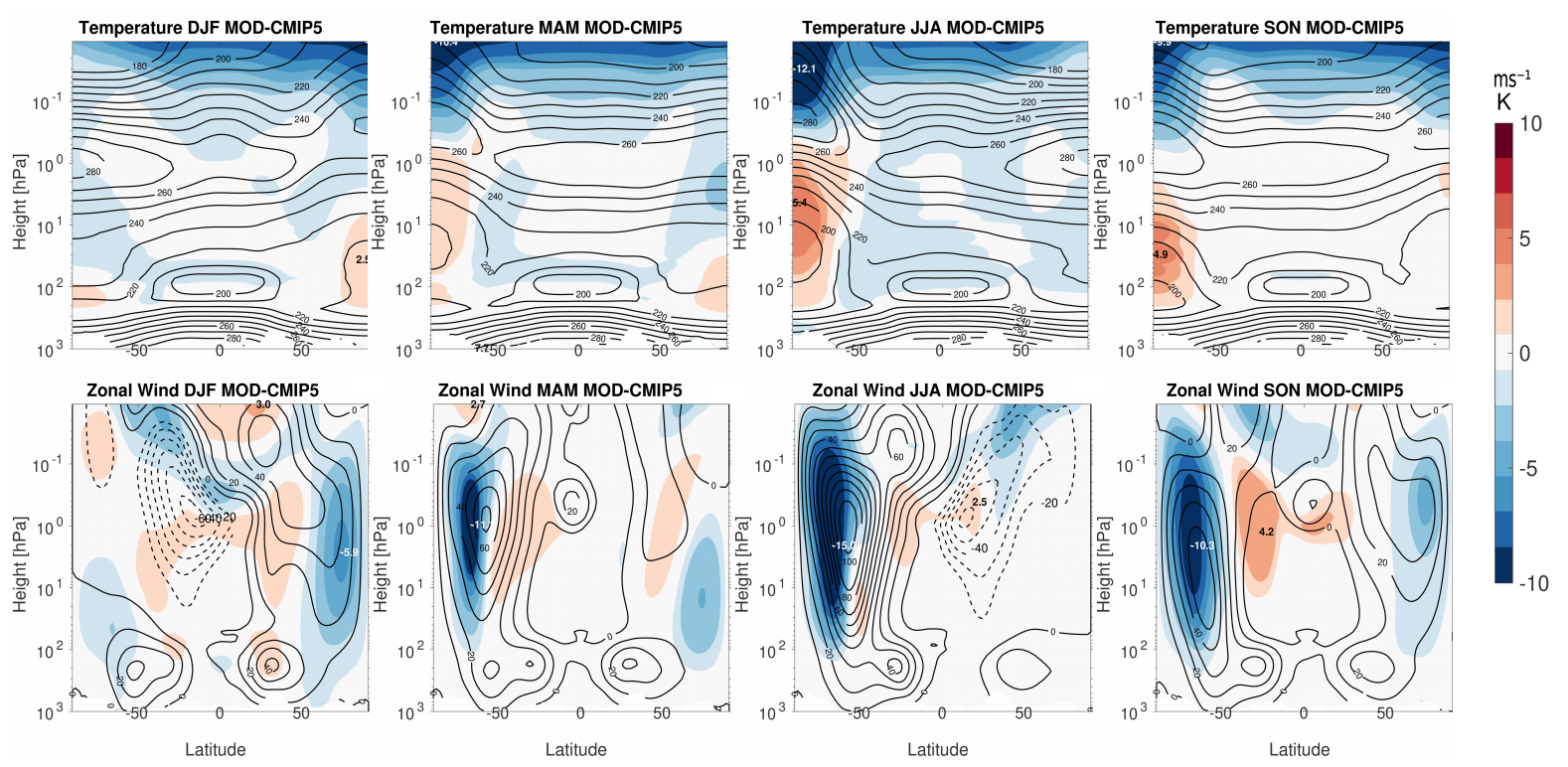

Figure 2: Seasonal mean difference between MOD and wMOD (shading) along with the mean for MOD (contours) for zonal mean temperature in $\mathrm{K}$ and zonal mean zonal wind in $\mathrm{m}^{-1}$ in a pre-industrial control simulation. MOD stands for model simulation with model code adjustments and wMOD stands for model simulation without model code adjustments. 

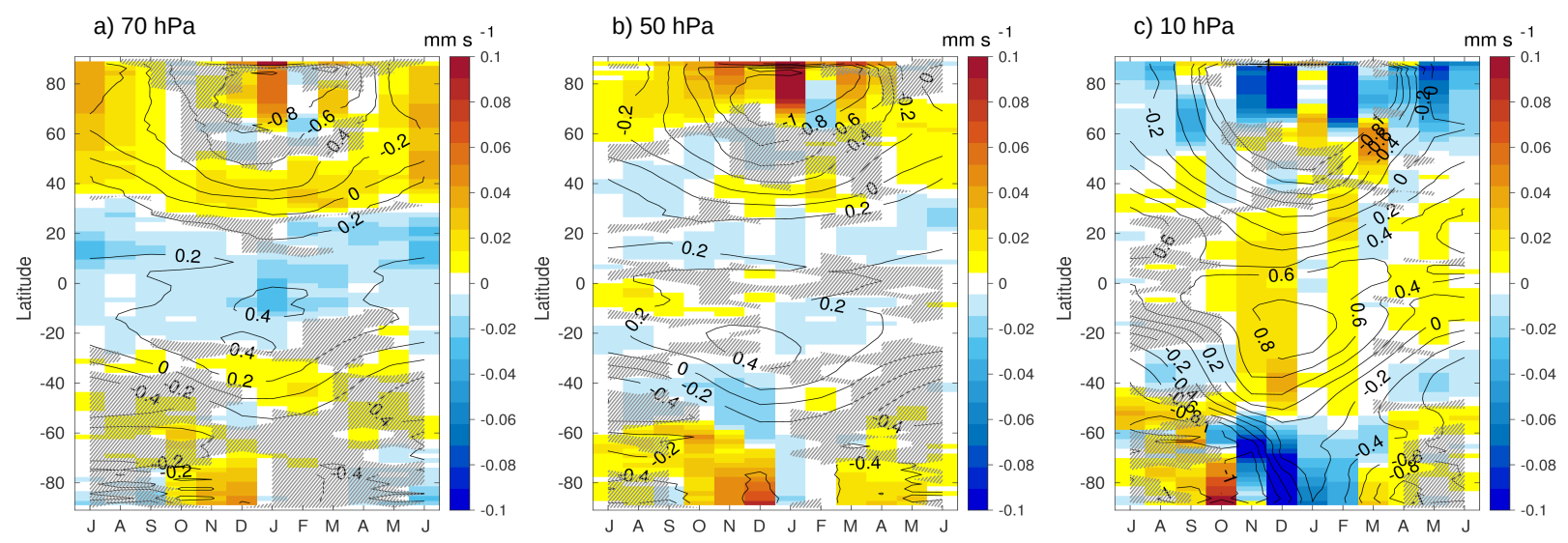

Figure 3: Monthly ensemble mean differences between Chem ON and Chem OFF for the vertical component of the meridional residual circulation $\mathrm{mm}^{-1}$ at $70 \mathrm{hPa}(\mathrm{a}), 50 \mathrm{hPa}$ (b), and $10 \mathrm{hPa}$ (c) as a function of latitude and month (shading). Contours represent the climatological mean state for Chem ON. The contour intervals are $0.2 \mathrm{mms}^{-1}$. Statistically insignificant regions are hatched at the $5 \%$ level based on a two-sided student's t-test. 

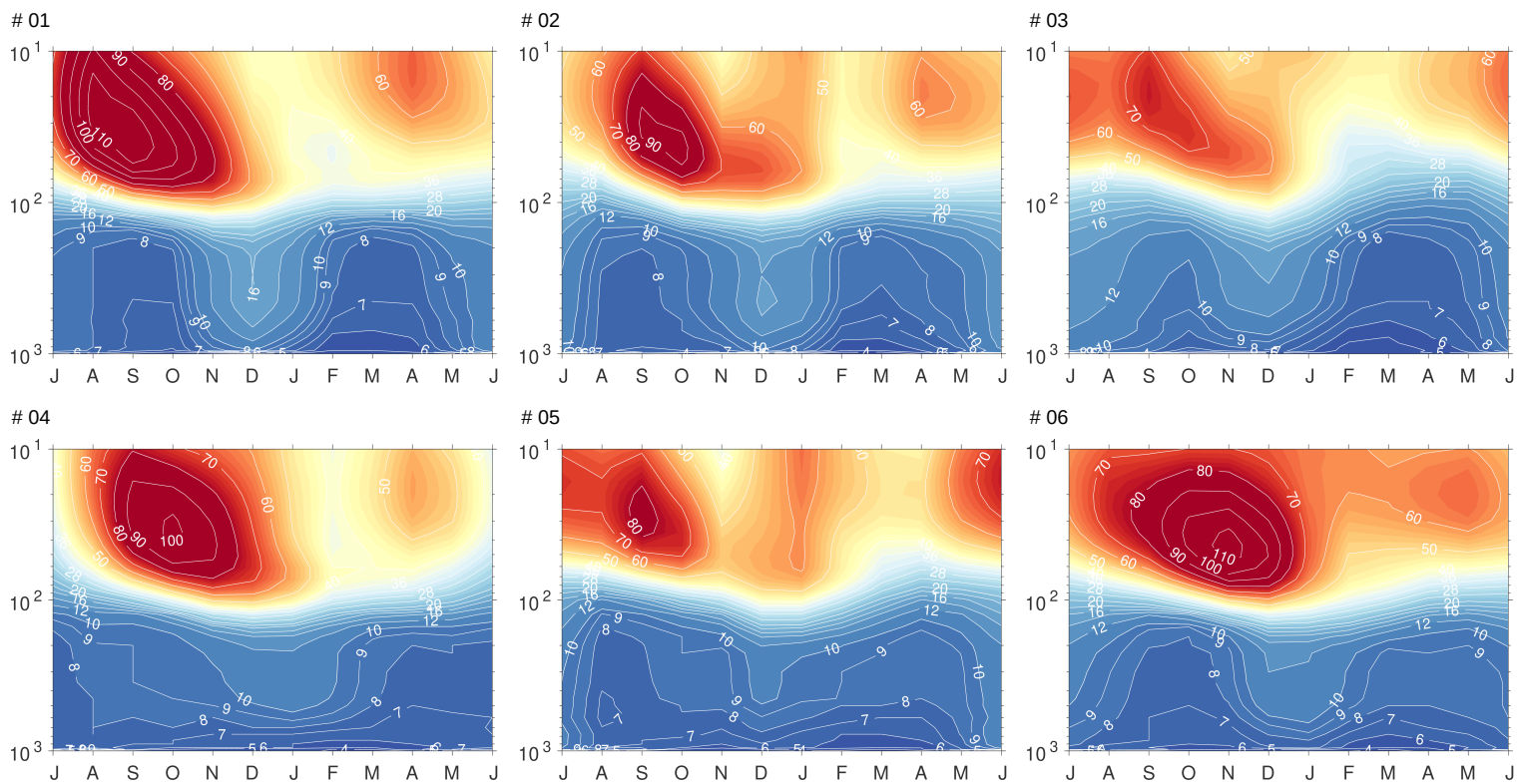
\# 05
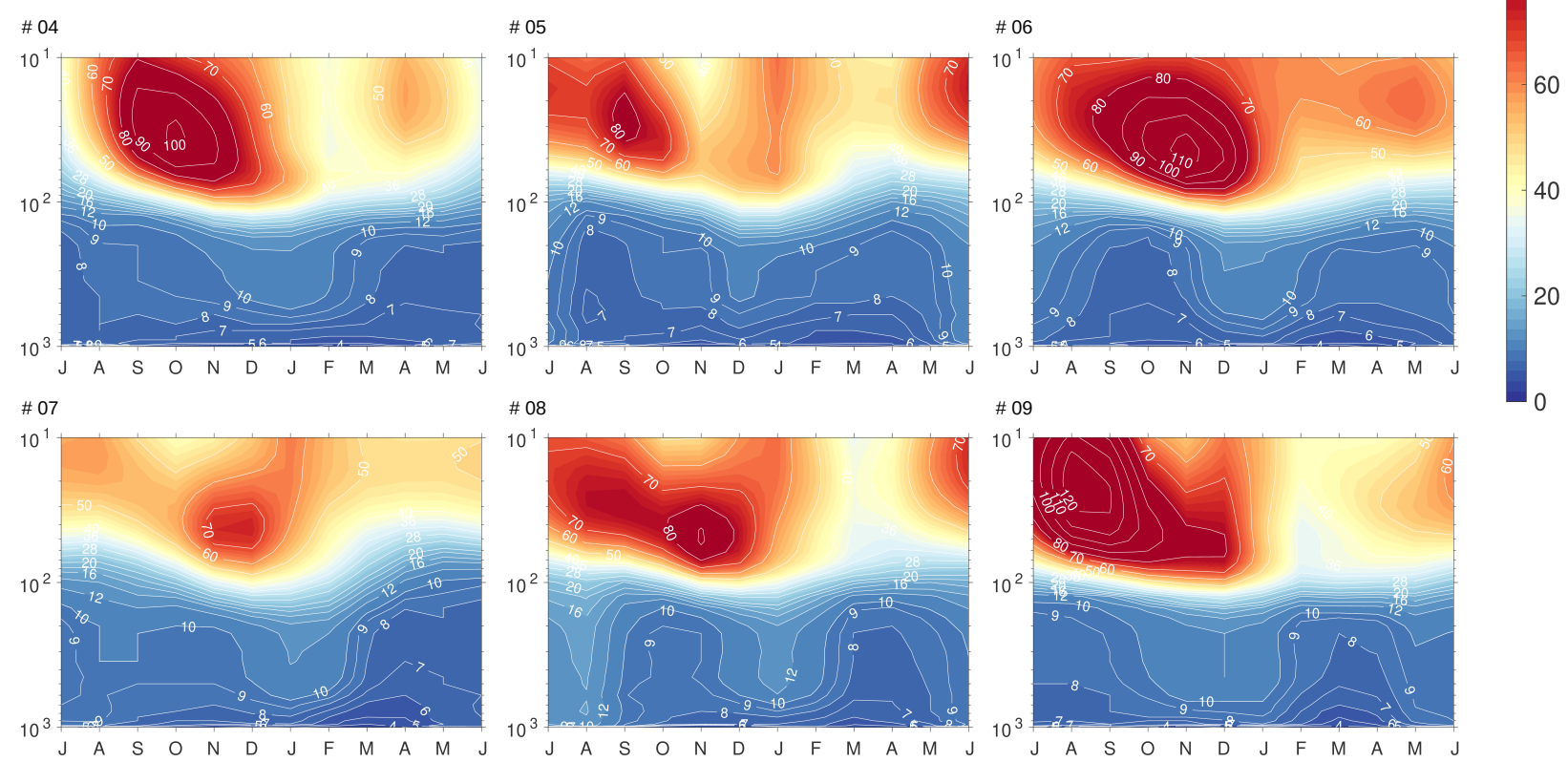

Figure 4: SAM timescale in days for the time period 1955-2013 for the individual ensemble members of Chem ON. 